\title{
VULNERABILIDADE, ESTRESSE, COVID-19 E COPING. UM ESTUDO COM CASAIS DE FAMÍLIAS DESFAVORECIDAS DA CIDADE DE QUELIMANE-MOÇAMBIQUE
}

\author{
VULNERABILITY, STRESS AND COVID-I9 AND COPING. A STUDY WITH \\ COUPLES FROM DISADVANTAGED FAMILIES IN THE CITY OF QUELIMANE- \\ MOZAMBIQUE
}

\section{Correia Hermenegildo Correia ${ }^{1}$ \\ Nédia Pereira Correia Mendes Correia ${ }^{2}$}

RESUMO: Este artigo traz uma discussão sobre vulnerabilidade, estresse, Covid-ı́ e estratégias de enfrentamento em casais de famílias desfavorecidas da cidade de Quelimane-Moçambique. A abordagem é qualitativa, assente na revisão bibliográfica de literaturas do tema em estudo. As fontes de investigação são o Google académico; Libgen; Mendeley Reference Maneger e Scielo. Os descritores usados foram vulnerabilidade, estresse, COVID-i9 e estratégias de enfrentamento "coping" e priorizou-se as publicações mais recentes possível. A discussão deste artigo toma como foco a influência da COVID-ı, em que coloca vários casais vulneráveis a contaminação e assume-se que as famílias desfavorecidas em particular, estejam expostos ao risco de contrair doenças, aspeto que faz com que vivam sempre ansiosos a procura da sustentabilidade para seus filhos por um lado e por outro pensando em si próprios e na saúde. Diante dos vários cenários relativos aos efeitos da COVID-I9 em que ocasiona e condiciona o mal-estar em casais e ou famílias da cidade de Quelimane, tendo em conta as particularidades e caraterísticas psicológicas procuram mobilizar diferentes estratégias cognitivas para se adaptar-se ao contexto e conclui-se que estas famílias mesmos atravessando momentos de crises elas assumem os riscos e os desafios levando a vida com maior naturalidade. Um outro dado interessante é que a cultura também influencia os estilos de enfrentamento das pessoas e, portanto, as intervenções devem abordar os pontos fortes, rituais e apoios dentro de uma comunidade. Portanto, as intervenções apropriadas precisam ser adaptadas à cultura e ao contexto em que os eventos traumáticos ocorrem (Carll, 2007).

Palavras-chave. Vulnerabilidade. Estresse.Covid-ı. Estratégias de enfrentamento. e Casais.

'Docente e Investigador da Universidade Licungo-Moçambique. Mestre em Psicologia Educacional pela Universidade Pedagógica de Moçambique e Doutorando em Psicologia Clínica pela Pontifícia Universidade Católica do Rio de Janeiro (PUC-RIO). E-mail: ccorreiahermenegildo@gmail.com.

${ }^{2}$ Docente e Investigadora da Universidade Licungo-Moçambique. Licenciada em Ensino de Biologia pela Universidade Pedagógica de Moçambique e Mestranda em Gestão Ambiental pela Universidade Licungo-Moçambique. 
ABSTRACT: This article discusses vulnerability, stress, Covid-r9 and coping strategies in couples from disadvantaged families in the city of QuelimaneMozambique. The approach is qualitative, based on a literature review of the subject under study. Research sources are academic Google; Libgen; Mendeley Reference Maneger and Scielo. The descriptors used were vulnerability, stress, COVID-r9 and coping strategies "coping" and the most recent publications possible were prioritized. The discussion of this article focuses on the influence of COVID-I9, which places several couples vulnerable to contamination and it is assumed that disadvantaged families in particular are exposed to the risk of contracting diseases, an aspect that makes them always anxious to looking for sustainability for their children on the one hand and thinking about themselves and their health on the other. In view of the various scenarios relating to the effects of COVID-I9 in which it causes and conditions malaise in couples and/or families in the city of Quelimane, taking into account the particularities and psychological characteristics, they seek to mobilize different cognitive strategies to adapt to the context and it is concluded that these families, even going through moments of crisis, they assume the risks and challenges, taking life more naturally. Another interesting fact is that culture also influences people's coping styles and, therefore, interventions must address strengths, rituals and supports within a community. Therefore, appropriate interventions need to be adapted to the culture and context in which traumatic events occur (Carll, 2007).

Keywords. Vulnerability. Stress. Covid-ı. Coping strategies. And Couples.

\section{INTRODUÇÃO}

A palavra vulnerável origina-se do latim vulnerare, que significa ferir, penetrar. Por essas razões etimológicas, vulnerabilidade é um termo geralmente usado na referência de predisposição a desordens ou suscetibilidade ao estresse (JANCZURA, 2012 p.302). Este conceito de vulnerabilidade não é novo, foi formulado nos anos 1930 por grupos de pesquisa de L.B. Murphy, que definiram o termo como "sustentabilidade à deterioração do funcionamento diante do estresse". Neste sentido, Murphy e seus colaboradores focaram suas pesquisas nas diferenças individuais das vulnerabilidades das crianças. Tal perspetiva acabou por reconhecer as complexas interações entre as vulnerabilidades, as forças individuais, o ambiente e a presença ou não de suporte social (YUNES \& SZYMANSKI, 200I p.28).

Nesta linha de pensamento, há que assumir que os primeiros trabalhos ancorados na perspetiva da vulnerabilidade social foram desenvolvidos, motivados 
pela preocupação de abordar de forma mais integral e completa não somente o fenômeno da pobreza, mas também as diversas modalidades de desvantagem social. Tais obras se destinaram a observar os riscos de mobilidade social descendente e as configurações vulneráveis que não se restringiram àqueles situados abaixo da linha de pobreza, mas a população em geral. Desta maneira, partiam do reconhecimento do fenômeno do bem-estar social de uma maneira dinâmica, bem como das múltiplas causas e dimensões associados a esse processo (ABRAMOVAY, 2002 p.28). A palavra vulnerabilidade insere-se no cotidiano atual, é reflexo de uma sociedade que se sente insegura e exposta ao perigo. Simultaneamente, o termo vem sendo usado das mais diversas formas e nos mais distintos contextos (vulneráveis no contexto da AIDS, crianças e jovens sem proteção da família, violência, exclusão social ou racial, ausência de privacidade, vulneráveis no contexto de desastres naturais e ou riscos socioambientais) (KUHNEN, 2009 p.39). Assim, a vulnerabilidade é definida como uma situação em que estão presentes três elementos: exposição ao risco; incapacidade de reação; e dificuldades de adaptação diante da materialização do risco (MOSER,1998). A discussão deste artigo toma como foco a influência da COVID-I9, em que coloca casais vulneráveis a contaminação e assume-se que as famílias desfavorecidas em particular, estejam expostos ao risco de contrair doenças, aspeto que faz com que vivam sempre ansiosos a procura da sustentabilidade para seus filhos por um lado e por outro pensando em si próprios e na saúde.

As teorias sobre o estresse $\mathrm{e}$ as formas de intervir no corpo-mente, consolidam e conduzem novos conceitos sobre como o estresse fisiológico (eustress) pode desencadear o estresse patológico (distress) no organismo (CANGUILHEM, 20II). Contudo, o estresse é um fenômeno normal no corpo humano. É o elemento desencadeador da motivação, da melhoria do desempenho em atividades e assume-se que seja o elemento que estimula a superação de desafios. Este é o eustress, estresse normal no nosso organismo funcionando como elemento fisiológico e propulsivo, impulsionador (SILVA, 2005). O COVID-ig sendo uma pandemia que esta causar mortes em vários países do mundo, e assumindo o seu impacto na vida dos cidadãos 
tais como histeria, fobia e desequilíbrio emocional, influencia inevitavelmente o estresse.

O COVID-ı9 é um vírus altamente infecioso e mortal, que está vitimando em quase todo o planeta terra. Teve o seu início em Dezembro de 2019, Wuhan província de Hubei na China (HEYMANN \& SHINDO, 2020). Aos poucos foi se propagando pelo mundo inteiro, causando vítimas mortais e simultaneamente paralisando a circulação das pessoas do mesmo contexto e de contextos diferentes, assim desacelerando a economia mundial. $O$ objetivo deste artigo é analisar a vulnerabilidade e estresse decorrentes da pandemia COVID-ı em casais de famílias moçambicanas, particularmente os da cidade de Quelimane, província da Zambézia. Moçambique fica situado no hemisfério Meridional entre os paralelos $10^{\circ} 27^{`}$ sul e $26^{\circ} 52^{`}$ sul. Ele pertence também ao Hemisfério Oriental entre os meridianos de $30^{\circ}$ I2 `Este e $40^{\circ} 51^{`}$ Oeste. O seu território enquadra-se no fuso horário dois, possuindo assim duas horas de avanço relativamente ao tempo Médio Universal, tal como uma parte dos países da Europa Setentrional e Oriental (MUCHANGOS, 1999 p.9).

No entanto, a província da Zambézia, situa-se na região centro de Moçambique. A sua capital é a cidade de Quelimane que é o foco do contexto deste artigo. Quelimane é a maior cidade e capital da província da Zambézia. Dada a sua localização geográfica, na zona costeira, a comercialização marítima e a pesca são uma das suas principais atividades econômicas. Mas há que sublinhar que a população vive da agricultura, cultivo de arroz, hortaliças e plantação do coqueiro (UCCLA, 2020). Estas caraterísticas socioculturais e económicas, associadas a COVID-ı, colocam a população em risco e vulnerável, uma vez que elas precisam sair para a rua procurar autossustento para si e suas famílias. Pesquisas recentes sobre a situação e evolução da pobreza em Moçambique, tanto pesquisas baseadas em dados estatisticamente representativos (ALKIRE \& SANTOS, 2oio; DE VLETTER et al., 2009; CUNGUARA \& HANLON, 2009), como pesquisas qualitativas, através de estudos de caso e reportagens narrativas (PAULO et al., 2008; SERRA, 2010), são unânimes num ponto: a pobreza continua muito elevada em Moçambique, 
cronicamente resistente e com sinais para aumentar, em vez de diminuir (CONCEIÇÃO, 2015 p. 273). Apesar de todos estes fatores juntos, a saúde esta em primeiro plano, e a melhor forma de evitar o contágio é ficar em casa. Este cenário é agonizante para quem não tem alternativas para a sobrevivência.

Esta análise é interessante e faz depreender o quanto as díades relacionais e dinâmicas sociais condicionam para a propagação ou não de determinadas doenças. Os casais vivem de incertezas, entre a pandemia da COVID-19 e a situação económica desfavorável fator que os impede de ficar em casa, e lutar pela sobrevivência, colocando em risco as suas vidas. Um dado, importante reside no fato de a Zambézia, com mais de quatro milhões de pessoas (25\% da população moçambicana), regista um forte agravamento da pobreza, tendo ultrapassado em 2009 o nível registado na primeira Avaliação da Pobreza, há mais de uma década (FRANCISCO, 2017). Neste sentido, faz-se necessário refletir algumas questões importantes: Em que medida a COVID-i9 condiciona a vulnerabilidade dos casais? Qual é o nível de preocupação dos casais face ao COVID-ıg? Que efeitos psicológicos pode causar a COVID-ı9? Que estratégias usam para enfrentar as adversidades da COVID-I9?

\section{MATERIAL E MÉTODO}

Este artigo constitui-se de uma abordagem qualitativa, assente na revisão bibliográfica de literaturas do tema em estudo. As fontes de investigação são o Google académico; Libgen; Mendeley Reference Maneger e Scielo. Os descritores usados foram vulnerabilidade, estresse, COVID-19 e estratégias de enfrentamento "coping" e priorizou-se as publicações mais recentes possível. Procurou-se usar artigos que discorressem sobre perspetiva da vulnerabilidade social, que foram desenvolvidos, motivados pela preocupação de abordar de forma mais integral e completa não somente o fenômeno da pobreza, mas também as diversas modalidades de desvantagem social. Trabalhos mais detalhados quanto ao estresse e as formas de intervir no corpo-mente, consolidam e conduzem novos conceitos sobre como o 
estresse fisiológico (eustress) pode desencadear o estresse patológico (distress) no individuo. Mais adiante, agregou-se trabalhos detalhados sobre a COVID-I9 e as estratégias de enfrentamento "coping".

\section{DISCUSSAO E RESULTADOS}

Os COVID-ı9 são uma família viral que causam infeções respiratórias e foram identificados um número de sete coronavírus humanos (HCOVs), nomeadamente: HCOV-229E, HCOV-OC43, HCOV-NL63, HCOV-HKUI, SARSCOV, MERS-COV, que causa síndrome respiratória do Oriente Médio e o SARSCOV (CHENG \& SHAN, 2020). O COVID-19 é transmitida por via de aspiro, de uma pessoa infetada para outra não infetada por via de pequenas gotículas do nariz ou boca que se espalham dentro de um contexto social (ORGANIZAÇÃO PANAMERICANA DA SAÚDE, 2020). Moçambique conta atualmente com 151367 casos positivos de COVID-I9, dentre estes, 149298 são dados como recuperados, I934 são óbitos e $\mathrm{I}$ I casos ativos. Estes dados resultam da última atualização feita no dia 06/II/202I pelo Ministério da Saúde de Moçambique, no âmbito das consequências socio sanitárias provocadas pela pandemia. No caso específico da cidade de Quelimane, província da Zambézia houve registo de II casos positivos de COVID-I9. Estes números resultam de uma serie de mecanismos de vigilância sanitária associada ao processo de vacinação em curso (GOVERNO DE MOÇAMBIQUE, 2021). Os dados aqui ilustrados, são politicamente coretos, mas vários são os cenários relativos a pandemia que não são divulgados.

Ao certo é que os casais de famílias desfavorecidas precisam sustentar os seus filhos, para tal devem se submeter ao risco ou a vulnerabilidade, o que estes casais mesmo com a pandemia em seu momento mais alto, não deixaram de sair a rua a procura de sobrevivência. $O$ estudo do enfrentamento ao estresse foi dividido em duas áreas: antecipação de eventos estressantes futuros e recuperação de traumas. Essas áreas foram mantidas notavelmente distintas na teoria e na pesquisa sobre enfrentamento. Embora existam diferenças claramente importantes entre os dois 
casos, optou-se por não enfatizar essa distinção. Portanto, para qualquer estresse, antecipação e recuperação nem sempre são claramente separáveis ou seja, quando estamos diante de uma situação estressante, a forma como agimos, em função das particularidades psicológicas individuais, dificilmente conseguimos perceber os atos distintos de evitar ou de recuperar; por conseguinte, lidar com um trauma envolve chegar a um acordo com o próprio evento e com a ameaça de recorrência no futuro (MANSELL, 2007). Seria útil se pudéssemos evitar todas as situações estressantes, mas isso é praticamente impossível. Todos enfrentamos pressão no cotidiano, por exemplo no trabalho, aborrecimentos diários, a morte de um membro da família e assim por diante. Como não podemos escapar do estresse, precisamos aprender a lidar efetivamente com nossos estressores (HUFFMAN et al., 1997 p.425).

Lazarus \& Folkman (1984) assumem o enfrentamento "coping" como esforços cognitivos e comportamentais em constante mudança para administrar as demandas externas e ou internas específicas que são avaliadas como excedendo o recurso da pessoa. Portanto, o enfrentamento ou coping compreende os mecanismos em que o indivíduo utiliza que lhe permitem lidar de forma adequada, ou não, com as situações indutoras de estresse (PEREIRA, 2006). Em termos mais simples, lidar é uma tentativa de gerenciar o estresse de alguma maneira eficaz. Não é um ato único, mas um processo que nos permite lidar com vários estressores. $O$ processo de enfrentamento pode se concentrar nos efeitos emocionais do estressor ou na solução do problema que causa o estresse (HUFFMAN et al., I997 p. 425). O enfrentamento está intimamente relacionado ao conceito de avaliação cognitiva e, portanto, nas transações pessoa-ambiente relevantes ao estresse e que o definem como "os esforços cognitivos e comportamentais feitos para dominar, tolerar ou reduzir demandas e conflitos externos e internos entre eles" (FOLKMAN \& LAZARUS, I989 p. 223).

Esta definição contém as seguintes implicações; (I) As ações de enfrentamento não são classificadas de acordo com seus efeitos (por exemplo, distorcem a realidade), mas de acordo com certas características do processo de enfrentamento. (2) Esse processo abrange reações comportamentais e cognitivas no 
indivíduo. (3) $\mathrm{Na}$ maioria dos casos, o enfrentamento consiste em diferentes atos únicos e é organizado sequencialmente, formando um episódio de enfrentamento. Nesse sentido, o enfrentamento é frequentemente caracterizado pela ocorrência simultânea de diferentes sequências de ações e, portanto, uma interconexão de episódios de enfrentamento. (4) As ações de enfrentamento podem ser distinguidas pelo foco em diferentes elementos de um encontro estressante (LAZARUS \& FOLKMAN, 1984). O que se pode observar é que os agentes estressores podem tentar mudar as realidades pessoa-ambiente por trás de emoções negativas ou estresse (enfrentamento focado no problema). Por outro lado, também podem se relacionar com elementos internos e tentar reduzir um estado emocional negativo ou alterar a avaliação da situação exigente, que seria um enfrentamento focado na emoção (MANSELL,2007). Contudo, é necessário assumir que a cultura também influencia os estilos de enfrentamento das pessoas e, portanto, as intervenções devem abordar os pontos fortes, rituais e apoios dentro de uma comunidade. Portanto, as intervenções apropriadas precisam ser adaptadas à cultura e ao contexto em que os eventos traumáticos ocorrem (Carll, 2007).

\section{CONCLUSÕES}

O COVID-ı9 é um problema de extrema relevância para a saúde pública em todo o mundo. Ela merece uma reflexão cuidadosa para contextos em que as populações são socioeconomicamente desfavorecidas. Faz se refletir que ficar em casa é uma medida adequada, mais ela se torna um caos, para quem sobrevive de pequenos negócios no cotidiano. O seu impacto na vida dos cidadãos em quase todo o mundo, é visível, está causando histeria, fobia e desequilíbrio emocional, contribuindo para o estresse. A OMS, o Ministério da Saúde de Moçambique, entre outras instituições, recomendam que os indivíduos devem evitar estar em lugares onde existe muita gente, lavar as mãos com água e sabão, higienizá-las com álcoolgel, usar máscaras naso-oral, cinza, evitar tocar os olhos, a boca e o nariz, se tiver alguma febre ou dificuldades respiratória, tosse, contactar ao centro de saúde 
próximo ou equipas médicas. São algumas medidas de evitamento da propagação do COVID-i9 (WORLD HEALTH ORGANIZATION \& MISAU Moçambique, 2020).

Estas medidas emanadas pelas autoridades de saúde são importantes mas difícil é cumpri-las, porque a maior parte da população/casais não tem condições e isto causa sentimentos de aceitar e não aceitar face as condições sociodemográficas dos tais casais, principalmente dos que ostentam baixa renda. Diante dos vários cenários relativos aos efeitos da COVID-19 em que ocasionam e condicionam o malestar em casais e ou famílias da cidade de Quelimane, tendo em conta as particularidades e caraterísticas psicológicas procuram mobilizar diferentes estratégias cognitivas para se adaptar-se ao contexto e conclui-se que estas famílias mesmos atravessando momentos de crises elas assumem os riscos e os desafios levando a vida com maior naturalidade, um outro dado interessante é que a cultura também influencia os estilos de enfrentamento das pessoas e, isso é notável nas diferentes formas como os casais de famílias desfavorecidas demonstram no cotidiano diante da pandemia.

\section{REFERENCIAS}

ABRAMOVAY, M; CASTro, M. G.; PINHEIRO, L. de C; LIMA, F. de S; MARTINELLI, C. da C. (2002) Juventude, Violência e Vulnerabilidade Social na América Latina: desafios para politicas políticas públicas. Brasília: UNESCO, BIO.

ALKIRE, S. e SANTOS, M.E. (2010) Acute Multidimensional Poverty: A New Index for developing Countries. OPHI Working Paper No. 38.

CANGUILHEM G. O. (2oII) normal e o patológico. $7^{\circ}$ ed. Forense Universitária. Rio de Janeiro (RJ).

CARLL. Elizabeth K. (2007). Trauma psychology: issues in violence, disaster, health, and illness. Editor Praeger Perspectives, Westport, Connecticut London. 
CHENG Z.J; SHAN J. (2020) Novel coronavirus: where we are and what we know. Vol. 48, Infection. Springer; p. 155-63.

CHICHANGO. I. P. A (2009). Geopolítica de Moçambique. Trabalho de investigação individual do CEMC 2008/o9. TCOR INF (RM). Lisboa.

CONCEIÇÃO. Francisco C. da. (2015). Implicações Politicas da Cooperação Internacional para o Desenvolvimento em Moçambique: da Sociedade à Trajetória Tradicional do Norte e à Experiência do Sul (1975-2013). Tese de doutorado, Programa de Pós-Graduação em Ciências Politicas, Universidade do Estado do Rio de Janeiro. CUNGUARA, B. \& HANLON, J.O (2010) Fracasso na Redução da Pobreza em Moçambique. Working Paper 74, Development as State-making. London: Crisis State Research Centre, LSE.

DE VASCONCElOS, L. (1999). Pela Boca Morre o Peixe. Associação dos Amigos de Leite de Vasconcelos. Maputo: PROMEDIA.

DE VletTer, F; LAUChANDE, C. e INFANTE, E. Fin. (2009). Scope Mozambique Survey - Survey Report. Maputo: AustralCowI.

GOVERNO DE MOÇAMBIQUE. Portal do Governo. (202I) Disponível em: http://www.portaldogoverno.gov.mz/por/Mocambique/Geografia-de-

Mocambique, acessado em 20 de Agosto.

HEYMANN D.L; SHINDO N. COVID-19 (2020): what is next for public health? Lancet. 22 de fevereiro ; 395(I0224):542-5.

HUFFMAN, Karen; VERNOY, Mark \& VERNOY, Judith (1997). Psychology in action. Editor, John Wiley \& Sons, Inc. New York.

INGC. Synthesis report. (2009). INGC Climate Change Report: Study on the impact of climate change on disaster risk in Mozambique. [van Logchem B and Brito R (ed.)]. INGC, Mozambique. 
JANCZURA, R.(2012). Risco ou Vulnerabilidade Social? Porto Alegre, Rs, Brasil, V.II, n.2

KUHNEN. A. (2009) Meio Ambiente e Vulnerabilidade: A Percepção Ambiental de Risco e o Comportamento Humano. Londrina v.I8 n.2

LAZARUS, R. S., \& FOLKMAN, S. (1984). Stress, appraisal, and coping. New York,

LAZARUS, R. S., \& FOLKMAN, (1989) Manual for the Study of Daily Hassles and Uplifts Scales. Palo Alto, CA: Consulting Psychologists Press.

MANSELL, W. (2007) Coping with fear and phobias. A step-by-step guide to understanding and facing you anxieties. Oneworld-Oxford, England.

MINISTÉRIO DA SAÚDE DE MOÇAMBIQUE (2020) [citado a 22 de Abril). $\begin{array}{llll}\text { Sobre } & \text { COVID-I9. } & \text { Retrieved } & \text { from }\end{array}$ http://www.misau.gov.mz/index.php/informacao-sobre-coronavirus-covid-I9 MOSER, C (1998). The asset vulnerability framework: reassessing urban poverty reduction strategies. World Development, New York, v.26, n.I.

MUCHANGOS, A. dos. (1999). Moçambique: Paisagens e Regiões, Tipografia Globo, Lda. República de Moçambique, oro48/FBM/93.

PAUlO, M; ROSÁRIO, C. \& TVEDTEN, I. (2008) Monitoria e Avaliação das Políticas de Redução da Pobreza em Moçambique Estudo 2: Relações Sociais da Pobreza Urbana.

SERRA, C. (2010). Linchamentos: moral não basta. . Várias reportagens, Disponível em: http://oficinadesociologia.blogspot.com/search?q=linchamentos.

SILVA J.J. (2005) Estresse: o impulso da vida. Yendis Editora; São Caetano do Sul $(\mathrm{SP})$.

UNIÃO DAS CIDADES CAPITAIS DE LÍNGUA PORTUGUESA (2020). Consultado 23.04, disponível em www.uccla.pt 
WORLD HEALTH ORGANIZATION. (2020) WHO announces COVID-i9 outbreak a pandemic. [citado 22 de abril de 2020];i. Available at: http://www.euro.who.int/en/health-topics/health-emergencies/coronavirus-covid19/news/news/2020/3/who-announces-covid-19-outbreak-a-pandemic.

YUNES, M.A.M e SZYMANSKI, H. (200I). Resiliência: noção, conceitos afins e considerações críticas in J. Tavares (Org.). Resiliência e educação, editora Cortez, São Paulo 